\title{
The Differences Among Attachment, Psychosocial Wellbeing and Academic Achievement Motivation of Foster Children and Their Socio-Demographic Variables in Save Our Soul Enfants Ethiope, Burayu Orphanage Vocational Training and Production Center (SOS EE BOVTPC)
}

\author{
Zenebe Demissie* \\ Psychology Department, Debire Birhan University University, PO box 247, Debire Brhan, Ethiopia \\ Habtamu Wondimu (Professor) \\ School of Psychology, Addis Ababa University, PO box 1121, Addis Ababa, Ethiopia
}

\begin{abstract}
The research is financed by the researchers themselves
Abstract

The general objective of this study was to explore the differences among attachment, psychosocial wellbeing and academic achievement motivation of foster children and their socio-demographic variables in SOS EE BOVTPC. Both quantitative and qualitative methods were employed to achieve the research objectives. The participants were: 134 foster children for quantitative methods and six foster families and six foster children for qualitative methods. The participants were selected using stratified random sampling technique, while the six foster families and the six foster children were purposefully taken as a sample. The self-report version of the Relationship Structures (ECR-RS) questionnaire, the Strengths and Difficulties Questionnaire (SDQ) for children age ranging above 11, the Achievement Motivation Inventory (AMI), and semi-structured interviews instruments were administered. Data from the quantitative survey were analyzed using mean score, standard deviation, independent t-test, and one way ANOVA. The qualitative data were analyzed using inductive thematic analysis. Independent T-test for group mean difference revealed that there was no significant difference in female and male foster children attachment, psychosocial wellbeing, and academic achievement motivation. On the other hand, there were significant difference in task orientation and anxious attachment of foster children who live with married and single parents. ANOVA result indicated that, the avoidance attachment shows a significant difference in the educational levels of foster parents. However, there were no differences in the overall psychosocial wellbeing of foster children and foster families educational levels. Nonetheless, the overall academic achievement motivation has shown significant differences between each educational level of foster children. This indicated that the organization should give emphasize for the improvement of their weakness.
\end{abstract}

Keywords: Academic Achievement Motivation, Attachment, Psychosocial Wellbeing

DOI: $10.7176 / \mathrm{NMMC} / 94-01$

Publication date: December $31^{\text {st }} 2020$

\section{Introduction}

Attachment defined in different ways. It refers to the strong emotional bond that exists between an infant and his/her caretaker and also defined as relationship between two people and forms the basis for long-term relationships (Roy and Kathleen 2007). Sensitive and responsive parenting promotes a secure attachment relationship (Nievar \& Becker, 2008), which in turn is predicatively associated with positive out-comes in numerous domains of child development (Dexter et al., 2013). Children raised only by the mother show poorer outcomes in several areas of development (levels of social emotional adaptation, social competence, cognitive scores, school achievements as well as higher rates of anxiety disorders and deviant behaviors Choe et al., (2013). Studies on attachment relationship between single mothers and their children showed inconsistent results ranging from increased to slightly decreased children's attachment security (Shaw et al., 2012). In addition to this poor educational level of parents affect their attachment with foster children (Seow, 2012).

Psychosocial well-being is the positive age-and stage-appropriate, outcome of children's physical, social and psychological development. It is essential for children's survival and development, especially in chronically difficult circumstances (Linda, Geoff \& Lorraine, 2006). Research finding based on psychosocial wellbeing revealed the difference between male and female. According to Perez (2012) females have significantly higher scores in the aspects of daily spiritual experience, relationship with father, relationship with peer, positive relationship with others and purpose in life. Males in other side have higher scores on autonomy than their female counterparty (Perez, 2012). Another research finding revealed that, educational level of foster children and parents have made differences on foster children psychosocial wellbeing's (Zabibu, 2012 and John et al, 2013).

Academic achievement motivation is used to mean the pupil's need or drive towards the achievement of 
success in academic work (Muola, 2010). A variety of factors in a youth's micro system can affect their educational achievement motivation, including family structure (e.g., living in a single-parent family), low parental involvement, neighborhood social disorganization, and low family socio economic status (Alexandra, 2009). Parent's educational levels have also an impact on children academic achievement (Yonzewa, 2000).

Studying on the differences among attachment, psychosocial wellbeing and academic achievement motivation of foster children and their socio-demographic variables has unaccountable benefit for stakeholders. So, this study intended to examine the differences among attachment, psychosocial wellbeing and academic achievement motivation of foster children and their socio-demographic variables in SOS Enfants Ethiope, Burayu Orphanage Vocational Training and Production Center.

\section{Research Questions}

1. To what extant dose attachment, psychosocial wellbeing and academic achievement motivation of foster children vary by socio-demographic variables?

\section{Methods and Materials \\ Description of the study area}

The study was conducted in Save Our Soul Enfants Ethiope, Burayu Orphanage Vocational Training and Production Center (SOS EE BOVTPC). SOS Enfantes Ethiope (SOSEE) is an international charitable organization which was founded by the two benevolent French nationals, Mr. Yves Ferez and M/Rs Jaquelin Ferez 27 years ago in Ethiopia. Currently, the organization is found in the Oromia region, Burayu-Keta woreda; which is near to Addis Ababa. The alternative cares (i.e. especially foster cares and community cares) are practiced in the then community, which found around the place where the organization is found.

\section{Research design}

To investigate the differences among attachment, psychosocial wellbeing and academic achievement motivation of foster children and their socio-demographic variables in SOS EE BOVTPC, the mixed (quantitative and qualitative) research design was employed.

\section{Population}

The current study targeted children who are between 15-18 years old who live with foster families with the support of SOS EE BOVTPC. There are 240 children in the foster care systems who, are considered as the study population. Stratified random sampling was used to select sample representative from the whole population. The sample size for this study was determined by using Yamane's (1967) formula. $\mathrm{n}=\frac{N}{1+N(e) 2}$ Where $\mathrm{N}$ is the size of the population and $\mathrm{n}$ is the size of the sample, $\mathrm{e}$ is the level of precision, 0.05 alpha level is the context of the present study. The computed sample size for this study was 134 foster children.

\section{Measures}

The researcher adopted Fraley et al, (2011) The Relationship Structures (ECR-RS) Questionnaire instrument to measure the attachment relationship of foster children with foster families of SOS EE BOVTPC. It is a self-report instrument designed to assess attachment patterns in a variety of close relationships. The items are used to assess attachment styles concerning 4 targets (i.e., mother, father, romantic partner, and best friend). Each item is rated on a 7 -point scale where $1=$ strongly disagree and $7=$ strongly agree. The test-retest reliability of the individual scales is approximately.65 for the domain of romantic relationships and.80 in the parent domain.

Besides, to identify the psychosocial wellbeing of foster children Goodman, (1997) Strengths and Difficulties Questionnaire (SDQ) scale was adopted which consisted of 25 questions. It is a behavioral screening tool, designed for children that measure psychosocial well-being across five dimensions: (1) emotional symptoms, (2) conduct problems, (3) hyperactivity/inattention, (4) peer relationship problems, and (5) pro-social behavior. Each sub-scale has 5 items, scored on a 3 -point Likert scale $(0=$ Not True, $1=$ Somewhat True, and $2=$ Certainly True $)$. The validity of the self-reported total difficulties scale has been assessed and confirmed in multiple contexts (Cornbrash's alpha ranging from $0.73-0.89$ ), indicating that the scale itself is internally valid.

To assess the academic achievement motivation of foster children the researcher adopted the Achievement Motivation Inventory (AMI). It can measure overall motivation toward academic achievement through a 20 -item inventory that takes approximately 15 minutes to complete. Each item has five response categories: "strongly agree," "agree," "not certain," "disagree," and "strongly disagree." The instrument has four scales (self-efficacy, control, task orientation, task value) and reliability coefficient (internal consistency) was .84, as measured using Cornbrash's alpha. For both three instruments, the Amharic version is translated by the researcher himself in collaboration with English language experts (i.e. MA holder in linguistic and litterateur).

A qualitative semi-structured interview was designed and used to explore the differences among attachment, 
psychosocial wellbeing and academic achievement motivation of foster children and their socio-demographic variables. The aim is to allow the respondents to inform the study from their point of view, using their own words and to get supporting idea for the quantitative data. A semi-structured interview guide consisting of themes and questions were prepared in English and then administered in Amharic. The Amharic version is translated by the researcher himself in collaboration with English language experts (i.e. MA holder in linguistic and litterateur).

\section{Data Analysis}

The collected data were organized, tabulated and analyzed by using percentage, mean, standard deviation, and ANOVA. For the analysis of the qualitative data, the researcher relied on inductive thematic analysis.

\section{Ethical Consideration}

Participants gave informed consent for participation in the study.

\section{Results}

Demographic Characteristics of the Foster Children and Foster Parents

In this part of the study the statistical result of foster children's sexes and educational level and foster families' marital status and educational levels are presented.

Table 1. Demographic Characteristic of Foster Children and Foster Families $(N=134)$

\begin{tabular}{llll}
\hline Demographic Variables & & $\mathbf{N}$ & $\mathbf{\%}$ \\
\hline Foster Children Sex & Male & 77 & 57.5 \\
& Female & 57 & 42.5 \\
\hline Foster Children Educational Levels & $1^{\text {st }}$ Level & 19 & 14.2 \\
& $2^{\text {nd }}$ Level & 47 & 35.1 \\
& Preparatory & 33 & 24.6 \\
\hline Foster Families Marital Status & TVT \& College & 35 & 26.1 \\
\hline Foster Families Educational Levels & Single & 79 & 59.0 \\
& Married & 55 & 41.0 \\
\hline & $1^{\text {st }}$ Level & 67 & 50.0 \\
& $2^{\text {nd Level }}$ & 32 & 23.0 \\
& Preparatory & 21 & 15.7 \\
\hline
\end{tabular}

Table 1 indicates that 77(57.5\%) were males and 57(42.5\%) were females. For those foster children $19(14.2 \%)$ had $1^{\text {st }}$ level education, $47(35.1 \%)$ had $2^{\text {nd }}$ level education, 33(24.6\%) were preparatory students, and $35(26.1 \%)$ had a higher education. Whereas 79(59\%) foster parents are single and 55(41\%) are married parents. Among those parents, $67(50 \%)$ had $1^{\text {st }}$ level education, $32(23 \%)$ had $2^{\text {nd }}$ level, $21(15.7 \%)$ had preparatory and $14(10.4 \%)$ had a higher education. In the present study the minimum score of foster children age is 15 and maximum $20(M=17.79$; $S D=1.44)$, length of stay (in years) in foster home minimum score 1 and maximum $12(M=4.67 ; S D=2.55)$.

Difference in Dependent Variables with Respect to Foster Children's Sex and Foster Parents' Marital Status Table 2. Summary of the Independent T-Test for Foster Children's Sex Difference $(N=134)$

\begin{tabular}{|c|c|c|c|c|c|}
\hline Variables & Sex & $\mathbf{N}$ & Mean & Std. Deviation & Sig. \\
\hline \multirow[t]{2}{*}{ Emotional Symptom } & Male & 77 & 3.44 & 2.06 & \multirow[t]{2}{*}{.80} \\
\hline & Female & 57 & 3.92 & 2.09 & \\
\hline \multirow[t]{2}{*}{ Conduct Problems } & Male & 77 & 3.44 & 1.87 & \multirow[t]{2}{*}{.93} \\
\hline & Female & 57 & 3.78 & 1.86 & \\
\hline \multirow[t]{2}{*}{ Hyperactivity } & Male & 77 & 4.75 & 1.30 & \multirow[t]{2}{*}{.34} \\
\hline & Female & 57 & 5.08 & 1.57 & \\
\hline \multirow[t]{2}{*}{ Peer Problems } & Male & 77 & 3.77 & 1.26 & \multirow[t]{2}{*}{.01} \\
\hline & Female & 57 & 3.80 & 1.79 & \\
\hline \multirow[t]{2}{*}{ Pro-social Behaviors } & Male & 77 & 6.22 & 2.39 & \multirow[t]{2}{*}{.24} \\
\hline & Female & 57 & 6.35 & 2.11 & \\
\hline \multirow[t]{2}{*}{ Total PSWB } & Male & 77 & 15.41 & 4.16 & \multirow[t]{2}{*}{.11} \\
\hline & Female & 57 & 16.61 & 4.71 & \\
\hline \multirow[t]{2}{*}{ Self-efficacy } & Male & 77 & 20.84 & 3.34 & \multirow[t]{2}{*}{.51} \\
\hline & Female & 57 & 20.15 & 3.35 & \\
\hline \multirow[t]{2}{*}{ Control } & Male & 77 & 18.59 & 3.52 & \multirow[t]{2}{*}{.16} \\
\hline & Female & 57 & 17.85 & 4.29 & \\
\hline
\end{tabular}




\begin{tabular}{llllll}
\hline Variables & Sex & N & Mean & Std. Deviation & Sig. \\
\hline Task Orientation & Male & 77 & 17.01 & 3.28 & .58 \\
& Female & 57 & 17.50 & 3.41 & .72 \\
\hline Task Value & Male & 77 & 17.70 & 3.28 & .68 \\
& Female & 57 & 17.29 & 2.95 & .18 \\
\hline Total AAM & Male & 77 & 74.15 & 8.76 & .17 \\
\hline Avoidance & Female & 57 & 72.82 & 10.31 & 5.19 \\
\hline Anxiety & Male & 77 & 25.87 & 6.63 & \\
& Female & 57 & 23.38 & 4.33 & 3.64 \\
\hline
\end{tabular}

As table 2 depicted that an independent-samples $t$ - test comparing the mean scores of the male and female children. Among the overall variables peer problem, found a significant difference between the means of the two groups $(t(132)=.01, p<.05)$. The mean score of the female children $(M=3.80, S D=1.79)$ was significantly higher than the mean of male children $(M=3.77, S D=1.26)$. Female children have higher peer problems than male. This implies that female foster children have border line symptoms on their peer problems. And also an independent samples $t$ - test was calculated comparing the mean score of total PSWB, AAM, Avoidance Attachment and Anxious Attachment. The result indicated that no significant difference was found in the total $\operatorname{PSWB}(t(132)=.11, p>.05)$. The mean of the male children $(M=15.41, S D=4.16)$ was not significantly different from the mean of female children $(M=16.61, S D=4.71)$, and the total AAM $(t(132)=.68, p>.05)$. The mean of the male children $(M=74.15, S D=8.76)$ was not significantly different from the mean of female children $(M=$ 72.82, $S D=10.31)$.

Table3. Summary of the Independent T-test for Foster Families Marital Status $(N=134)$

\begin{tabular}{|c|c|c|c|c|c|}
\hline Variables & Marital Status & $\mathbf{N}$ & Mean & Std. Deviation & Sig. \\
\hline \multirow[t]{2}{*}{ Emotional Symptom } & Single & 79 & 3.58 & 2.02 & .46 \\
\hline & Married & 55 & 3.74 & 2.17 & \\
\hline \multirow[b]{2}{*}{ Conduct Problems } & Single & 79 & 3.43 & 1.81 & \\
\hline & Married & 55 & 3.81 & 1.94 & .64 \\
\hline \multirow[b]{2}{*}{ Hyperactivity } & Single & 79 & 4.96 & 1.50 & \\
\hline & Married & 55 & 4.80 & 1.32 & .25 \\
\hline \multirow[b]{2}{*}{ Peer Problems } & Single & 79 & 3.84 & 1.54 & \\
\hline & Married & 55 & 3.71 & 1.46 & .84 \\
\hline \multirow[b]{2}{*}{ Pro-social Behaviors } & Single & 79 & 6.37 & 2.13 & \\
\hline & Married & 55 & 6.12 & 2.46 & .20 \\
\hline \multirow[b]{2}{*}{ Total PSWB } & Single & 79 & 15.82 & 4.45 & \\
\hline & Married & 55 & 16.07 & 4.43 & .67 \\
\hline \multirow[b]{2}{*}{ Self-efficacy } & Single & 79 & 21.07 & 3.26 & \\
\hline & Married & 55 & 19.80 & 3.37 & .48 \\
\hline \multirow[b]{2}{*}{ Control } & Single & 79 & 18.92 & 3.81 & \\
\hline & Married & 55 & 17.36 & 3.80 & .64 \\
\hline \multirow{3}{*}{ Task Orientation } & Single & 79 & 17.13 & 2.80 & \\
\hline & Married & 55 & 17.34 & 4.00 & .01 \\
\hline & Single & 79 & 17.12 & 2.81 & \\
\hline Task Value & Married & 55 & 18.10 & 3.50 & .12 \\
\hline \multirow{3}{*}{ Total AAM } & Single & 79 & 74.26 & 8.55 & \\
\hline & Married & 55 & 72.61 & 10.58 & .10 \\
\hline & Single & 79 & 25.81 & 5.35 & \\
\hline Avoidance & Married & 55 & 23.38 & 6.51 & .14 \\
\hline \multirow[b]{2}{*}{ Anxiety } & Single & 79 & 11.60 & 3.33 & \\
\hline & Married & 55 & 10.50 & 4.92 & .00 \\
\hline
\end{tabular}

As Table 3 depicted an independent-sample $t$ - test was comparing the mean scores of single and married parents. Among the overall variables, task orientation and anxiety of children who live in married and single families, found a significant difference between the means of the two groups $(t(132)=.01, p<.05)$ and $(t$ $(132)=.00, p<.05)$ respectively. The mean of the children task orientation who live with married parents $(M=17.34$, $S D=4.00)$ was significantly higher than the mean of single parents $(M=17.13, S D=2.80)$ and the mean of children's anxious attachment who live with single parents $(M=11.60, S D=3.33)$ was significantly higher than the mean of married parent $(M=10.50, S D=4.92)$. And also an independent-samples $t$ - test was calculated 
comparing the mean score of total PSWB \& AAM. The result indicated that no significant difference was found in the total PSWB $(t(132)=.67, p>.05)$. The mean of children who live with single parents $(M=15.82, S D=$ 4.45) was not significantly different from the mean of children who live with married parents $(M=16.07, S D$ $=4.43)$, and the total AAM $(t(132)=.10, p>.05)$. The mean of the single parents $(M=74.26, S D=8.55)$ was not significantly different from the mean of married parents $(M=72.61, S D=10.58)$.

Differences in Foster Children and Foster Families Educational Levels Differences in Foster Children Educational Levels

Table 4. ANOVA for Dependent Variables with Regards to Children Educational Levels $(N=134)$

\begin{tabular}{|c|c|c|c|c|c|c|}
\hline Variables & Groups & Sum of Squares & df. & Mean Square & F. & Sig. \\
\hline \multirow[t]{3}{*}{ Total PSWB } & Between Groups & 60.39 & 3 & 20.13 & 1.02 & .38 \\
\hline & Within Groups & 2550.86 & 130 & 19.62 & & \\
\hline & Total & 2611.25 & 133 & & & \\
\hline \multirow[t]{3}{*}{ Total AAM } & Between Groups & 353.10 & 3 & 117.70 & 1.33 & .26 \\
\hline & Within Groups & 11501.32 & 130 & 88.47 & & \\
\hline & Total & 11854.42 & 133 & & & \\
\hline \multirow[t]{3}{*}{ Avoidance Attachment } & Between Groups & 168.91 & 3 & 56.30 & 1.60 & .19 \\
\hline & Within Groups & 4551.41 & 130 & 35.01 & & \\
\hline & Total & 4720.33 & 133 & & & \\
\hline \multirow[t]{3}{*}{ Anxious Attachment } & Between Groups & 43.50 & 3 & 14.50 & .86 & .45 \\
\hline & Within Groups & 2170.20 & 130 & 16.69 & & \\
\hline & Total & 2213.70 & 133 & & & \\
\hline
\end{tabular}

As Table 4 indicated that there is no significant difference among the variables $F(3,130)=1.02, p=.38, p>.05$, $F(3,130)=1.33, p=.26, p>.05), F(3,130)=1.60, p=.19, p>.05$ and $F(3,130)=.86, p=.45, p>.05$ respectively. The children at the four educational levels did not differ significantly in all total PSWB, AAM, avoidance attachment, and anxious attachment. So there is no need to refer to the "Multiple Comparisons" table. However, there is a significant difference among the children educational levels of their peer problem. Table $9 \& 10$ depict these results.

Table 5. ANOVA for Peer Problems with Regards to Children Educational Levels

\begin{tabular}{lllllll}
\hline Variables & Groups & Sum of Square & Df. & Mean Square & F & Sig. \\
\hline Peer Problems & Between Groups & 22.34 & 3 & 7.45 & 3.46 & .02 \\
& Within Groups & 279.80 & 130 & 2.15 & & \\
& Total & 302.14 & 133 & & & \\
\hline
\end{tabular}

As Table 5 depicted there was a statistical significance difference between children educational levels on their peer problems $F(3,130)=3.46$; $p=.02$. So it is necessary to show "Multiple Comparisons" to identify the difference between foster children educational levels on peer problems.

Table 6. Multiple Comparisons: Dependent Variable: Peer Problem

\begin{tabular}{|c|c|c|c|c|c|c|}
\hline \multirow[t]{2}{*}{$\begin{array}{l}\text { (I)Educational } \\
\text { Levels of Children }\end{array}$} & \multirow{2}{*}{$\begin{array}{l}\text { (J) Educational } \\
\text { Levels of } \\
\text { Children }\end{array}$} & \multirow[t]{2}{*}{$\begin{array}{l}\text { Mean Difference } \\
\text { (I-J) }\end{array}$} & \multirow[t]{2}{*}{$\begin{array}{l}\text { Std. } \\
\text { Error }\end{array}$} & \multirow[t]{2}{*}{ Sig. } & \multirow{2}{*}{\begin{tabular}{|l|}
$95 \%$ \\
Interval \\
Lower \\
Bound \\
\end{tabular}} & \multirow{2}{*}{$\begin{array}{c}\text { Confidence } \\
\text { Upper } \\
\text { Bound } \\
\end{array}$} \\
\hline & & & & & & \\
\hline \multirow[t]{4}{*}{ First Level } & Second Level & -.75 & .39 & .24 & -1.78 & .28 \\
\hline & Preparatory & .28 & .42 & .90 & -.81 & 1.38 \\
\hline & TVT \& College & -.27 & .41 & .91 & -1.36 & .81 \\
\hline & First Level & .75 & .39 & .24 & -.28 & 1.78 \\
\hline \multirow[t]{3}{*}{ Second Level } & Preparatory & $1.03^{*}$ & .33 & .01 & .16 & 1.90 \\
\hline & TVT \& College & .47 & .32 & .46 & -.37 & 1.32 \\
\hline & First level & -.28 & .42 & .90 & -1.38 & .81 \\
\hline \multirow[t]{3}{*}{ Preparatory } & Second level & $-1.03^{*}$ & .33 & .01 & -1.90 & -.16 \\
\hline & TVT \& College & -.55 & .35 & .40 & -1.48 & .36 \\
\hline & First level & .27 & .41 & .91 & -.81 & 1.36 \\
\hline \multirow[t]{2}{*}{ TVT \& College } & Second level & -.47 & .32 & .46 & -1.32 & .37 \\
\hline & Preparatory & .55 & .35 & .40 & -.36 & 1.48 \\
\hline
\end{tabular}

*. The mean difference is significant at the 0.05 level.

Tukey's HSD was used to determine the nature of the differences between the children's educational levels. This analysis revealed that children who had a preparatory level of education were lower peer problems $(\mathrm{M}=3.24$, $\mathrm{SD}=1.52)$ than children who had a secondary level of education $(\mathrm{M}=4.27, \mathrm{SD}=1.36)$. This implies that foster 
children who have second level of education have border line symptoms on their peer problems. Children who had a $1^{\text {st }}$ level $(M=3.52, S D=1.71)$ and children who had a TVT \& College level $(\mathrm{M}=3.80, \mathrm{SD}=1.41)$ were not significantly different from either of the other two groups regarding their peer problems.

Differences in Foster Parents Educational Levels

Table 7. ANOVA for Dependent Variables with Regards to Parents Educational Levels $(N=134)$

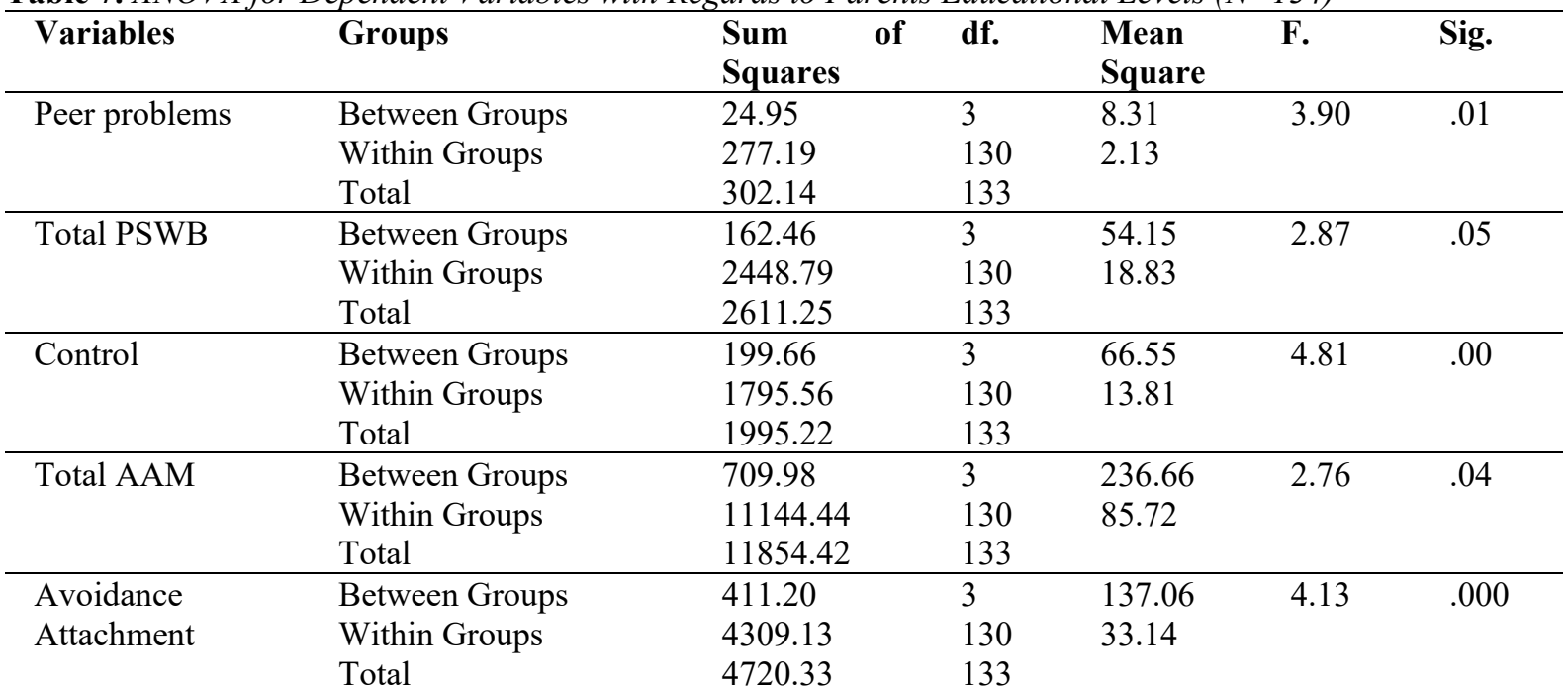

As Table 7 depicted among the sub-scales of PSWB, peer problem result indicated that there is significant difference, $(F(3,130)=3.90, p=.01, p<.05$. On the other hand, among the sub-scales of AAM, control result indicated that there is a significant difference, $F(3,130)=4.81, p=.00, p<.05$ and the total AAM result indicated that there is a significant difference, $F(3,130)=2.76, p=.04$. From the two attachment relation sub-scales avoidance attachment result indicated that there is significant difference, $(F(3,130)=4.13, p=.00, p<.05$. The children who live with parents who have four educational levels are different in their PSWB, AAM, and AR. So referring to the multiple comparison tables is necessary to determine the nature of the differences between the educational levels of parents.

Table 8. Multiple Comparisons: Dependent Variable: Peer Problem: Tukey HSD

\begin{tabular}{|c|c|c|c|c|c|c|}
\hline \multirow{2}{*}{$\begin{array}{l}\text { (I)Educational } \\
\text { Level of Parents }\end{array}$} & \multirow{2}{*}{$\begin{array}{l}(J) \quad \text { Educational } \\
\text { Level of Parents }\end{array}$} & \multirow{2}{*}{$\begin{array}{l}\text { Mean } \\
\text { Difference } \\
\text { (I-J) }\end{array}$} & \multirow{2}{*}{$\begin{array}{l}\text { Std. } \\
\text { Error }\end{array}$} & \multirow[t]{2}{*}{ Sig. } & \multicolumn{2}{|c|}{ 95\%Confidence Interval } \\
\hline & & & & & $\begin{array}{l}\text { Lower } \\
\text { Bound }\end{array}$ & Upper Bound \\
\hline \multirow[t]{4}{*}{ First Level } & Second Level & .29 & .31 & .77 & -.51 & 1.11 \\
\hline & Preparatory & -.78 & .36 & .14 & -1.73 & .16 \\
\hline & Higher Education & -.92 & .42 & .13 & -2.04 & .18 \\
\hline & First Level & -.29 & .31 & .77 & -1.11 & .51 \\
\hline \multirow[t]{3}{*}{ Second Level } & Preparatory & $-1.08^{*}$ & .41 & .04 & -2.15 & -.01 \\
\hline & Higher Education & $-1.22^{*}$ & .46 & .04 & -2.44 & -.00 \\
\hline & First Level & .78 & .36 & .14 & -.16 & 1.73 \\
\hline \multirow[t]{3}{*}{ Preparatory } & Second Level & $1.08^{*}$ & .41 & .04 & .01 & 2.15 \\
\hline & Higher Education & -.14 & .50 & .99 & -1.45 & 1.16 \\
\hline & First Level & .92 & .42 & .13 & -.18 & 2.04 \\
\hline \multirow[t]{2}{*}{ Higher Education } & Second Level & $1.22^{*}$ & .46 & .04 & .00 & 2.44 \\
\hline & Preparatory & .142 & .50 & .99 & -1.16 & 1.45 \\
\hline
\end{tabular}

*. The mean difference is significant at the 0.05 level.

Tukey's $H S D$ was used to determine the nature of the differences between children peer problems, who live with parents who had different educational levels. As Table 8 revealed that children who live with parents who had higher education levels have higher scores on peer problems $(M=4.57, S D=1.74)$ than second levels $(M=$ $3.34, S D=1.53$ ), and children who live with parents who had a preparatory level of education have higher score on peer problems $(M=4.42, S D=1.69)$ than parents who had a secondary level of education $(M=3.34, S D=$ 1.53). This implies that foster children have border line symptoms on their peer problems. Children who live with parents who had $1^{\text {st }}$ level education $(M=3.64, S D=1.27)$ were not significantly different from any of the other three levels. 
Table 9. Multiple Comparisons: Dependent Variable: Control Tukey HSD

\begin{tabular}{|c|c|c|c|c|c|c|}
\hline \multirow[t]{2}{*}{$\begin{array}{l}\text { (I)Educational Level of } \\
\text { Parents }\end{array}$} & \multirow[t]{2}{*}{$\begin{array}{l}\text { (J) Educational Level of } \\
\text { Parents }\end{array}$} & \multirow{2}{*}{$\begin{array}{l}\text { Mean } \\
\text { Difference } \\
\text { (I-J) }\end{array}$} & \multirow[t]{2}{*}{$\begin{array}{l}\text { Std. } \\
\text { Error }\end{array}$} & \multirow[t]{2}{*}{ Sig. } & \multicolumn{2}{|c|}{$\begin{array}{l}95 \% \text { Confidence } \\
\text { Interval }\end{array}$} \\
\hline & & & & & $\begin{array}{l}\text { Lower } \\
\text { Bound }\end{array}$ & $\begin{array}{l}\text { Upper } \\
\text { Bound }\end{array}$ \\
\hline \multirow[t]{4}{*}{ First Level } & Second Level & .81 & .79 & .73 & -1.26 & 2.89 \\
\hline & Preparatory & -2.40 & .92 & .05 & -4.81 & .01 \\
\hline & Higher Education & 2.02 & 1.09 & .25 & -.81 & 4.87 \\
\hline & First Level & -.81 & .79 & .73 & -2.89 & 1.26 \\
\hline \multirow[t]{3}{*}{ Second Level } & Preparatory & $-3.21^{*}$ & 1.04 & .01 & -5.93 & -.49 \\
\hline & Higher Education & 1.21 & 1.19 & .73 & -1.88 & 4.31 \\
\hline & First Level & 2.40 & .92 & .05 & -.01 & 4.81 \\
\hline \multirow[t]{3}{*}{ Preparatory } & Second Level & $3.21^{*}$ & 1.04 & .01 & .49 & 5.93 \\
\hline & Higher Education & $4.42^{*}$ & 1.28 & .00 & 1.09 & 7.76 \\
\hline & First Level & -2.02 & 1.09 & .25 & -4.87 & .81 \\
\hline \multirow{2}{*}{ Higher Education } & Second Level & -1.21 & 1.19 & .73 & -4.31 & 1.88 \\
\hline & Preparatory & $-4.42^{*}$ & 1.28 & .00 & -7.76 & -1.09 \\
\hline
\end{tabular}

*. The mean difference is significant at the 0.05 level.

Tukey's $H S D$ was used to determine the nature of the differences between children control behavior, who live with parents' who had different educational levels. Table 9 revealed that children control behavior, who live with parents who had a preparatory level of education have higher score $(M=20.71, S D=3.01)$ than parents who had a secondary level of education $(M=17.50, S D=3.63)$ and higher educational levels $(M=16.23, S D=4.41)$. Children control behavior, who live with parents who had $1^{\text {st }}$ level education $(M=18.36, S D=3.79)$ were not significantly different from any of the other three levels.

Table 10. Multiple Comparisons: Dependent Variable: Total AAM

\begin{tabular}{|c|c|c|c|c|c|c|}
\hline \multirow[t]{2}{*}{$\begin{array}{l}\text { (I)Educational Level of } \\
\text { Parents }\end{array}$} & \multirow[t]{2}{*}{$\begin{array}{l}\text { (J) Educational Level of } \\
\text { Parents }\end{array}$} & \multirow{2}{*}{$\begin{array}{l}\text { Mean } \\
\text { Difference } \\
\text { (I-J) }\end{array}$} & \multirow[t]{2}{*}{$\begin{array}{l}\text { Std. } \\
\text { Error }\end{array}$} & \multirow[t]{2}{*}{ Sig. } & \multicolumn{2}{|c|}{$\begin{array}{l}95 \% \text { Confidence } \\
\text { Interval }\end{array}$} \\
\hline & & & & & $\begin{array}{l}\text { Lower } \\
\text { Bound }\end{array}$ & $\begin{array}{l}\text { Upper } \\
\text { Bound }\end{array}$ \\
\hline \multirow[t]{4}{*}{ First level } & Second Level & -.36 & 1.98 & .99 & -5.53 & 4.81 \\
\hline & Preparatory & -4.21 & 2.31 & .26 & -10.23 & 1.81 \\
\hline & Higher Education & 4.92 & 2.72 & .27 & -.2 .156 & 12.01 \\
\hline & First Level & .36 & 1.98 & .99 & -4.81 & 5.53 \\
\hline \multirow[t]{3}{*}{ Second level } & Preparatory & -3.85 & 2.60 & .45 & -10.62 & 2.91 \\
\hline & Higher education & 5.29 & 2.96 & .28 & -2.43 & 13.01 \\
\hline & First Level & 4.21 & 2.31 & .26 & -1.81 & 10.23 \\
\hline \multirow[t]{3}{*}{ Preparatory } & Second Level & 3.85 & 2.60 & .45 & -2.91 & 10.62 \\
\hline & Higher education & $9.14^{*}$ & 3.19 & .03 & .82 & 17.45 \\
\hline & First Level & -4.92 & 2.72 & .27 & -12.01 & 2.15 \\
\hline \multirow[t]{2}{*}{ Higher education } & Second Level & -5.29 & 2.96 & .288 & -13.01 & 2.43 \\
\hline & Preparatory & $-9.14^{*}$ & 3.19 & .03 & -17.45 & -.82 \\
\hline
\end{tabular}

*. The mean difference is significant at the 0.05 level.

Tukey's HSD was used to determine the nature of the differences between children AAM, who live with parent's who had different educational levels. As Table 10 depicted that children AAM, who live with parents who had a preparatory level of education have higher score $(M=77.57, S D=7.76)$ than parents who had higher levels of education $(M=68.42, S D=14.34)$. Children's AAM, who live with parents who had $1^{\text {st }}$ level education $(M=$ $73.35, S D=9.13)$ and $2^{\text {nd }}$ levels $(M=73.71, S D=7.52)$ were not significantly different from any of the other three levels. This implies that parents who have preparatory educational levels give regular support to their foster children to score high AAM than parents who have higher educational levels. 
Table 11. Multiple Comparisons: Dependent Variable: Avoidance Attachment

\begin{tabular}{|c|c|c|c|c|c|c|}
\hline \multirow[t]{2}{*}{$\begin{array}{l}\text { (I)Educational Level } \\
\text { of Parents }\end{array}$} & \multirow[t]{2}{*}{$\begin{array}{l}\text { (J) Educational Level } \\
\text { of Parents }\end{array}$} & \multirow{2}{*}{$\begin{array}{l}\text { Mean } \\
\text { Difference } \\
\text { (I-J) }\end{array}$} & \multirow[t]{2}{*}{$\begin{array}{l}\text { Std. } \\
\text { Error }\end{array}$} & \multirow[t]{2}{*}{ Sig. } & $\begin{array}{l}95 \% \\
\text { interval }\end{array}$ & confidence \\
\hline & & & & & $\begin{array}{l}\text { Lower } \\
\text { Bound }\end{array}$ & $\begin{array}{l}\text { Upper } \\
\text { Bound }\end{array}$ \\
\hline \multirow[t]{4}{*}{ First level } & Second Level & 4. $08^{*}$ & 1.23 & .00 & .86 & 7.30 \\
\hline & Preparatory & 2.82 & 1.43 & .20 & -.92 & 6.57 \\
\hline & Higher Education & 2.49 & 1.69 & .45 & -1.91 & 6.89 \\
\hline & First Level & $-4.08^{*}$ & 1.23 & .00 & -7.30 & -.86 \\
\hline \multirow[t]{3}{*}{ Second level } & Preparatory & -1.26 & 1.61 & .86 & -5.46 & 2.94 \\
\hline & Higher education & -1.59 & 1.84 & .82 & -6.39 & 3.20 \\
\hline & First Level & -2.82 & 1.43 & .20 & -6.57 & .92 \\
\hline \multirow[t]{3}{*}{ Preparatory } & Second Level & 1.26 & 1.61 & .86 & -2.94 & 5.46 \\
\hline & Higher education & -.33 & 1.98 & .99 & -5.50 & 4.83 \\
\hline & First Level & -2.49 & 1.69 & .45 & -6.89 & 1.91 \\
\hline \multirow[t]{2}{*}{ Higher education } & Second Level & 1.59 & 1.84 & .82 & -3.20 & 6.39 \\
\hline & Preparatory & .33 & 1.98 & .99 & -4.83 & 5.50 \\
\hline
\end{tabular}

*. The mean difference is significant at the 0.05 level.

Tukey's HSD was used to determine the nature of the differences between children's avoidance attachment, who live with parent's who had different educational levels. Table 11 revealed that foster children, who live with parents who had a $1^{\text {st }}$ level of education have higher score on their avoidance attachment $(M=26.49, S D=5.09)$ than parents who had a secondary level of education $(M=22.40, S D=6.89)$ and higher educational levels $(M=$ 16.23, $S D=4.41)$. This implies that foster children who live with parents who have first level education have unsecured relationship than parents who have second levels of education or their relationship may be full of discomfort/distrust. Children, who live with parents who had a preparatory level of education $(M=23.66, S D=$ $5.41)$ and higher educational levels $(M=24.00, S D=6.43)$ were not significantly different from each other on their avoidance attachment.

Interviewees revealed that, any attachment problems, lack of psychosocial wellbeing, and insufficient academic achievement occur because of different reasons; among these reasons lack of intimate relationship, unequal treatment between their biological child and foster child, gender biasness, illiterateness or poor education level of foster families, marital irregularity (single or married), blame shifting, etc. This lead foster children to attachment problems like:- being avoidance (fearful avoidant and dismissal avoidant), being anxious, quarreling with their foster family, being lone loss, etc. Psychosocial problems like:- emotional problems (lack of emotional intelligence), conduct problems (immorality), lack of discipline, hopelessness, loneliness, emotional disturbance (hyperactivity), developing antisocial behavior, disrespectful, aimlessness/lack of explicated objective, etc.; academic achievement problems like:- lack of self-efficacy, absenteeism from regular class, lack of motivation to study, not doing their class work and homework properly, reducing their ranks, repetition in one class for several times. The absence of restrict follow-up, reinforcement, appreciation, motivation, encouragement, etc. leads to the irregularity of academic achievement motivation of foster children.

\section{Discussion}

Difference in Dependent Variables with Respect to Foster Children Sex and Foster Parents Marital Status The anxious attachment result indicated that foster children who live with single parents have higher anxious attachment than foster children who live with married foster parents. This implies that foster children who live with single parents have no secured attachment than foster children who live with married parents. Single foster parents may have different problems to make secured attachment relationships with foster children. This finding is consistent with the finding of Shaw et al. (2012). In this study; children raised in single mother show poorer outcomes in several areas of development when compared to two-parent families (Shaw et al., 2012).

The result revealed that among the sub- scales of the overall psychosocial wellbeing, peer problem result showed significant difference between the two sexes (i.e. peer problems of female foster children are greater than the male foster children). This implies that female foster children have a border line symptom on their peer problems than male foster children. In the other variables there is no significant difference between female and male foster children. This finding is inconsistent with the research done by Perez (2012), who revealed that females have significantly higher score in the aspects of peer relationship.

In the other part of the funding, among the sub-scales of academic achievement motivation, task orientation result indicated that there is a significant difference between married and single parents (i.e. foster children who live in married foster families are greater task orientation than single families). This indicated that foster children who live with married foster family are more concentrated on their academic tasks than foster children who live 
with single parents. Married foster family may support the children to focus on their academic tasks than domestic tasks. This result is consistent with the study by Alexandra (2009). According to Alexandra, youth in single-parent homes may also be disadvantaged in the way that they receive less parental supervision and monitoring of school work, and have less social capital than those who have married parents. The limited economic and social resources of many single parents are the primary cause of the lower educational achievement of youth from single-parent families compared to those of married parent families (Alexandra, 2009).

\section{Differences in Foster Children and Foster Families Educational Levels}

In this part of the study, the researcher investigated about the significant difference between foster children and foster family's educational levels and other variables. The result indicated that the avoidance attachments of foster children who live with parents who had a first level of education are higher than parents who had a secondary level of education and higher educational levels. On the other hand, avoidance attachment of foster children who live with parents who had preparatory and higher educational levels were not significantly different from each other. This implied that foster children have no secured relationship with parents who had a first level of education relatively than parents who had secondary and above educational levels. The result is consistent with Seow, (2012). According to him parents poor education conditions affecting parenting style and their attachment to their children.

The other variable result indicated that there is a difference in foster children peer problems among the four levels of education. Foster children who had second level education revealed higher peer problems than first level education. This implies that foster children who have second level education have borderline symptom on their peer problem. The other two (Preparatory and TVT \& College) had no differences. However, there is no significant difference among the other variables. As we know second level education (i.e. from grade nine \& ten) is considered as adolescent (puberty) stage. Theoretically this stage is identity or role confusion stage (Erik Erikson, 1950). According to him adolescent stage considered as a major stage in human development, if the adolescent, perform his/her role correctly at this stage to achieve the next stage with good manner; if not role confusion may occur. In another way adolescents at this stage search their identity in different status and occupations. Success in their identity leads them to make a good transition to the next stage. However, sometimes unsuccessfulness may occur in their life because of their identity crisis (Zabibu, 2012).

Foster children who live with parents who had higher educational levels have higher peer problems than who had a second level of education, and foster children who live with parents who had preparatory levels of education have higher peer problems than who had secondary educational levels. However, foster children who live with parents who had first educational levels have no significant differences with the other levels. The result indicated that the socialization processes of foster parents who had greater than secondary educational levels may be poorer than the primary educational levels. This result is inconsistent with the study of John et al. (2013); according to them parental education has its own effect on the psychosocial wellbeing of foster children. If the parent education level increased, particularly mothers' educational statuses have been linked to better behavioral and emotional adjustment in children. This indicated that at the time of selection should consider the educational status of the family (John et al, 2013).

On the other hand, the academic achievement motivations of foster children who live with parents who had preparatory levels of education are higher than parents who had higher educational levels. In the other levels there are no significant differences. The explanation of the result indicated that foster parents who had higher educational levels have not given concern to their foster children or they may be neglected their foster children directly or indirectly. This finding is supported by previous research findings, indicates that parent level of education was not significantly related to test scores in the foster care population (Alexandra, 2009). However; this result is inconsistent with the research of Yonzewa (2000). In line with Yonzewa, has shown that better-educated mothers are more likely to spend more time supervising their children and that better-educated parents are more likely to advocate for their child's placement in respect courses manage more actively in their child's academic achievement, and be more informed about school issues than less-educated parents.

The result of the qualitative data supported that, the differences among attachment, psychosocial wellbeing, and academic achievement motivation of foster children with their sex and educational level depend on the fostering and caring style of foster families (i.e. sense of humanity, control and follow up). The interviewee's idea is in line with the idea of parental sensitivity and control that was suggested by Fisher and Kim (2007). According to them, high emotional climate, including sensitive responses to children's needs revealed to be important, foster children having experienced adversity and unpredictability in their history seemed to need foster parents who additionally exert appropriate behavior-management. Monitoring and structuring might provide children with a secure base from which to explore and a clear framework that conveys a feeling of a safe and predictable environment (Fisher \& Kim, 2007).

\section{Conclusions}

Based on the major findings of the study, the following conclusions are drawn: The SOS EE BOVTPC fosters 
children attachment, psychosocial wellbeing, and academic achievement motivations are differ with their sex and educational levels. Those who live with single parents have higher anxious attachment. Female foster children have greater peer problem than the male foster children. Foster children who live in married foster families are greater task orientation than single families. Those who live with parents who had first level of education have higher avoidance attachments. Foster children who had second level education revealed higher peer problems. Those who live with parents who had preparatory and higher educational levels have higher peer problems. And also foster children who live with parents who had preparatory levels of education have higher academic achievement motivations.

\section{Recommendations}

The results of the study indicated that fosters children attachment, psychosocial wellbeing, and academic achievement motivations are differ with their sex and educational levels. To minimize and narrow the differences, psychosocial support or intervention should be implemented by professionals. Enhance the foster parent's educational level or be carful the level of education when placing children in foster parents.

It is better if SOS EE BOVTPC rearranges and organizes the service of guidance and counseling which plays a significant role in preventing, identifying and rehabilitating foster children who do have a lack of psychosocial wellbeing and foster parents who do have lack of attachment pattern.

In general, taking a comprehensive governmental and non-governmental approach to creating safe fostering environments by enhancing the full continuum of foster children's life services, and revising fostering policies as well as caregiving practices that influence communication and collaboration around these issues is good.

\section{Funding}

The current study was self-sponsored.

\section{Acknowledgments}

First and foremost, I would like to express my deepest gratitude to Professor Habtamu Wondimu my advisor, for his efforts in providing me with relevant advice, critical comments and constructive suggestion throughout my work. Second, I would like to thanks all staff, foster children, and foster families of SOS Enfants Ethiope, Burayu Orphanage Vocational Training and Production Center. Third, I am grateful to Ato Mitiku Hambisa for his invaluable comments and suggestions. Fourth, I would like to appreciate my sister Aster Demissie for her support, understanding and typing the paper.

\section{Conflict of interest}

I declared there is no conflict of interest with other authors and I assure that I am responsible for any conflict of interest that may arise.

\section{References}

Alexandra C. (2009). The effect of foster care experience and characteristics on academic achievement. Unpublished PhD Dissertation. University of North Carolina at Chapel Hill.

Choe, D.E.,Olson, S.L., and Sameroff, A.J. (2013). Effects of early maternal distress and parenting on the development of children's self-regulation and externalizing behavior. Dev.Psychopathol. 25, 2,437453.doi:10.1017/S095457941 2001162.

Dexter, C.A., Wong, K., Stacks, A.M., Beeghiy, M., and Barnett, D. (2013). Parenting and attachment among lowincome African-American and caucasian preschoolers. J.Fam.Psychol.27.629-638.

Erikson, E. (1950). Theory of psychosocial development.

Fraley, R. C., Heffernan, M. E., Vicary, A. M., \&Brumbaugh, C. C. (2011). The Experiences in close relationshipsrelationship structures questionnaire: A method for assessing attachment orientations across relationships. Psychological Assessment, 23, 615-625.

Goodman R. (1997). The strengths and difficulties questionnaire a research note. J Child Psychol Psychiatry, 38:581-586. DOI:10.1007/s00787-004-2005-3.

Gumede, RP. (2009). The psychosocial well-being of the teenaged orphans: Rural community Kwazulu-Natal, Unversity of South Africa, South Africa.

John G. Orme, Cheryl Buehler, Kathryn W. Rhodes, Mary Ellen Cox, Michael McSurdy, \& Gary Cuddeback (2013). Parental and familial characteristics used in the selection of foster families. University of Tennessee.

Linda Richter, Geoff Foster and Lorraine Sherr (2006). Where the heart is? Meeting the psychosocial needs of young children in the context of HIV/AIDS.

Muola J. M. (2010). A study of the relationship between academic achievement motivation and home environment among standard eight pupils.Educational Research and Reviews. 5 (5), pp. 213-217.

Nievar M.A. and Becker B.J. (2008). Sensitivity as a privileged predictor of attachment: A second perspective on 
De Wolff and Van IJzendoorn' smeta-analysis. Soc.Dev. 17,102-114.

Perez, P.M. (2012). An explanatory model of academic achievement based on aptitude, goal orientation, selfconcept and learning strategy. The Spanish Journal of Psychology, 15(1), 48-60.

Roy F. Baumeister \& Kathleen D. Vohs (2007). Encyclopedia of Social Psychology. SAGE Publications India Pvt. Ltd.

Seow, W.K., (2012). Environmental, Maternal, and Child factors which contribute to early childhood caries: A unifing conceptual model. Int.J.Paediatr.Den.22, 157-168.

Shaw, D., Hyde,L. V., and Brennan, L. L.(2012). Early predictors of boys' antisocial trajectories. Dev. Psychopathol. 24, 871-888.doi:10.1017/S0954579412000429.

Yamane, Taro. (1967). Statistics: An Introductory Analysis, $2^{\text {nd }}$ ED., New York: Harper and Row.

Yonezawa, S. (2000). Unpacking the black box of tracking decisions: Critical tales of families navigating the course of placement process. In M. G. Sanders (Ed.), Schooling students placed at risk: Research, policy, practice in the education of poor and minority adolescents (pp. 109-140). Mahwah, NJ: Erlbaum.

Zabibu Khamis Mbangwa (2012). Assessment of psychosocial well-being of adolescent orphans aged 13-15 years old: A case study of Upendo orphanage centre in Moshi Municipality. open University of Tanzania. 